\title{
Robust Adaptive Dynamic Surface Control for a Class of Nonlinear Dynamical Systems with Unknown Hysteresis
}

\author{
Yong-Hua Liu,, ${ }^{1,2}$ Ying Feng, ${ }^{1,2}$ and Xinkai Chen ${ }^{3}$ \\ ${ }^{1}$ The Key Laboratory of Autonomous System and Network Control, Ministry of Education, China \\ ${ }^{2}$ The College of Automation Science and Engineering, South China University of Technology, Guangzhou 510640, China \\ ${ }^{3}$ The Department of Electronic and Information Systems, Shibaura Institute of Technology, 307 Fukasaku, \\ Minuma-ku, Saitama, Saitama 337-8570, Japan
}

Correspondence should be addressed to Ying Feng; zhdfengying@gmail.com

Received 17 June 2013; Accepted 19 November 2013; Published 2 January 2014

Academic Editor: Massimo Furi

Copyright (C) 2014 Yong-Hua Liu et al. This is an open access article distributed under the Creative Commons Attribution License, which permits unrestricted use, distribution, and reproduction in any medium, provided the original work is properly cited.

\begin{abstract}
The output tracking problem for a class of uncertain strict-feedback nonlinear systems with unknown Duhem hysteresis input is investigated. In order to handle the undesirable effects caused by unknown hysteresis, the properties in respect to Duhem model are used to decompose it as a nonlinear smooth term and a nonlinear bounded "disturbance-like" term, which makes it possible to deal with the unknown hysteresis without constructing inverse in the controller design. By combining robust control and dynamic surface control technique, an adaptive controller is proposed in this paper to avoid "the explosion complexity" in the standard backstepping design procedure. The negative effects caused by the unknown hysteresis can be mitigated effectively, and the semiglobal uniform ultimate boundedness of all the signals in the closed-loop system is obtained. The effectiveness of the proposed scheme is validated through a simulation example.
\end{abstract}

\section{Introduction}

With the development of smart materials, some smart materials-based actuators, such as piezoceramic actuators [1], magnetostrictive actuators, and shape memory alloys, are becoming increasingly important in the application areas of aerospace, manufacturing, defense, and civil infrastructure systems [2-5], because of their excellent performance, for example, high precision, fast response, and flexible actuating ability [6-8]. However, a class of nonsmooth nonlinearities, hystereses, with multibranching and nondifferential properties, widely occur in these smart materials-based actuators. When the system is preceded by these actuators, the existence of the hysteresis behaviour in these actuators will degrade the system performance, causing undesirable inaccuracy. The hysteresis nonlinearities are the nature properties of these smart materials, which cannot be cancelled by the improvement of the smart materials. Therefore, how to mitigate the negative effects caused by the hysteresis nonlinearities from control view becomes one important research topic in this area. Due to the nonsmooth nature of hysteresis, most common control approaches developed for nonlinear systems may not be applicable to hysteretic systems directly, which attracted significant attention in the modeling of hysteresis nonlinearities and the hysteretic systems controller design.

For the modeling method of the hysteresis, it can be roughly classified as differential equation-based hysteresis models, such as Backlash-like model [9], Bouc-Wen model $[10,11]$, and Duhem model $[10,12]$, and operator-based hysteresis models, such as Preisach model, Krasnosel'skiiPokrovskii model, and Prandtl-Ishlinskii model [13-15]. As a differential-equation based hysteresis model, Duhem model can represent numerous hysteresis shapes including saturation and asymmetric properties by choosing different shape functions. However, the output analytical expression of Duhem model is difficult to obtain directly since the output depends on the solution of the differential equation, which may cause a new difficulty for the controller design.

So far, the control design work for the systems in presence of hysteresis nonlinearities has also been paid more attention [16-19]. Generally, two control approaches are used to 
mitigate the negative effects of hysteresis in the literature. The common one is to construct a hysteresis inverse model to cancel the adverse effects of hysteresis completely or approximately, such as $[20,21]$. The main advantage of this inverse control approach is to compensate the effects of hysteresis nonlinearities directly. However, the construction of the inverse hysteresis will increase the complexity of the control systems and may limit the application in the industrial systems. Also, the compensation error depends on the hysteresis modeling parameters; therefore, it is difficult to get the analytical expression of the compensation error. Alternatively, another method is to fuse the hysteresis models with control methods without constructing the hysteresis inverse $[9,22-24]$, which can be applied in the real-time systems conveniently. For this control structure without inverse, the key point is to explore the characteristics of the hysteresis model and then investigate the suitable control methods to mitigate the effects caused by hysteresis.

Synthesizing the hysteresis modeling methods and control approaches, the output tracking problem for a class of uncertain nonlinear systems in strict-feedback form with unknown Duhem hysteresis is discussed. For the Duhem model, one adaptive robust controller for a class of nonlinear systems was discussed in [25]. Still following the line, the robust adaptive control method for a class of uncertain nonlinear systems in strict-feedback form is investigated in this paper. In order to mitigate the design difficulty caused by the smooth function term in the uncertain nonlinear systems, the mean value theorem and a Nussbaum function lemma are used. The proposed dynamic surface control (DSC) approach [26] without hysteresis inverse avoids "the explosion complexity" in the standard backstepping design, mitigates the negative effects arising from the unknown hysteresis, and ensures the semiglobal uniform ultimate boundedness of all the signals in the closed-loop system.

The rest of this paper is organized as follows. In Section 2, the control problem is formulated. Duhem hysteresis model is introduced in Section 3. In Section 4, an adaptive dynamic surface controller is developed for a class of nonlinear systems in strict-feedback form with unknown Duhem hysteresis, and the stability analysis is given as well. Computer simulations are shown to verify the effectiveness of the proposed scheme in Section 5. Section 6 concludes the paper.

\section{Problem Statement}

Consider the following class of uncertain nonlinear systems in strict-feedback form with unknown hysteresis input:

$$
\begin{gathered}
\dot{x}_{i}=\theta_{i} f_{i}\left(\bar{x}_{i}\right)+g_{i} x_{i+1}+d_{i}(x, t), \quad i=1, \ldots, n-1, \\
\dot{x}_{n}=\theta_{n} f_{n}(x)+g_{n} w(u)+d_{n}(x, t), \\
y=x_{1},
\end{gathered}
$$

where $\bar{x}_{i}=\left[x_{i}, \ldots, x_{i}\right]^{T} \in R^{i}, i=1, \ldots, n ; x=\bar{x}_{n}=$ $\left[x_{1}, \ldots, x_{n}\right]^{T} \in R^{n}$ and $y \in R$ are the system states and output; $g_{i}, \theta_{i}$ are unknown constant parameters, $d_{i}(x, t)$ denote the unknown disturbances, $f_{i}(\cdot)$ are known smooth functions, $i=1, \ldots, n$; and $w \in R$ is the output of the hysteresis nonlinearity with the actual input $u$.

The control objective is to design a control law $u$ in (1), forcing the output $y$ to track a given desired trajectory $y_{d}$, while all the signals of closed-loop system are uniformly bounded.

The following assumptions of the system (1) are made.

Assumption 1. The desired trajectory $y_{d}$ is continuous and its first-order derivative $\dot{y}_{d}$ and second-order derivative $\ddot{y}_{d}$ are bounded and available; that is, there exists a positive constant $B_{0}$, such that $\Omega_{d}=\left\{\left[y_{d}, \dot{y}_{d}, \ddot{y}_{d}\right]^{T}: y_{d}^{2}+\dot{y}_{d}^{2}+\ddot{y}_{d}^{2} \leq B_{0}\right\} \subset R^{3}$.

Assumption 2. The signs of $g_{i}$ are known, and there exist unknown positive constants $g_{i 0}$ and $g_{i 1}$ such that $0<g_{i 0} \leq$ $\left|g_{i}\right| \leq g_{i 1}<\infty$. Without loss of generality, it can be assumed that $0<g_{i 0} \leq g_{i}, i=1, \ldots, n$.

Assumption 3. The disturbances $d_{i}(x, t), i=1, \ldots, n$, satisfy

$$
\left|d_{i}(x, t)\right| \leq b_{i} \rho_{i}\left(\bar{x}_{i}\right),
$$

where $\rho_{i}\left(\bar{x}_{i}\right)$ are known nonnegative smooth functions and $b_{i}$ are unknown nonnegative constants.

Remark 4. It should be mentioned that the knowledge of $g_{i 0}$ and $g_{i 1}$ is not required to be known, which is only used in the analysis of the latter stability proof.

\section{Hysteresis Model}

In this paper, the Duhem model is used to describe the hysteresis nonlinearity, which is defined by [14]

$$
\frac{d w}{d t}=\alpha\left|\frac{d u}{d t}\right|(\lambda(u)-w)+\frac{d u}{d t} \psi(u),
$$

where $u$ and $w$ are the hysteresis input and output, respectively; $\alpha$ is a constant; and $\lambda(u)$ and $\psi(u)$ are shape functions of $u$.

In order to get the analytic expression of the hysteresis output $w$, the following three conditions $[10,27,28]$ are used for Duhem model.

Condition 1. $\lambda(u)$ is a piecewise smooth, monotone increasing, odd function of $u$, with a derivative $\dot{\lambda}(u)$, that obtains a finite $\operatorname{limit}_{\lim _{u \rightarrow \infty}} \dot{\lambda}(u)$.

Condition 2. $\psi(u)$ is a piecewise continuous, even function of $u$, with a finite limit satisfying

$$
\lim _{u \rightarrow \infty} \psi(u)=\lim _{u \rightarrow \infty} \dot{\lambda}(u) .
$$

Condition 3. $\dot{\lambda}(u)>\psi(u)>\alpha e^{\alpha u} \int_{u}^{\infty}|\dot{\lambda}(\zeta)-\psi(\zeta)| e^{-\alpha \zeta} d \zeta$ for all finite $u$.

Remark 5. By selecting suitable shape functions, Duhem model can describe the different characteristics of the hysteresis nonlinearities. For example, choose $\alpha=5$ and $\psi(u)=$ $\dot{\lambda}(u)\left(1-0.85 e^{(-0.1|u|)}\right)$ with different shape function $\lambda(u)$ 


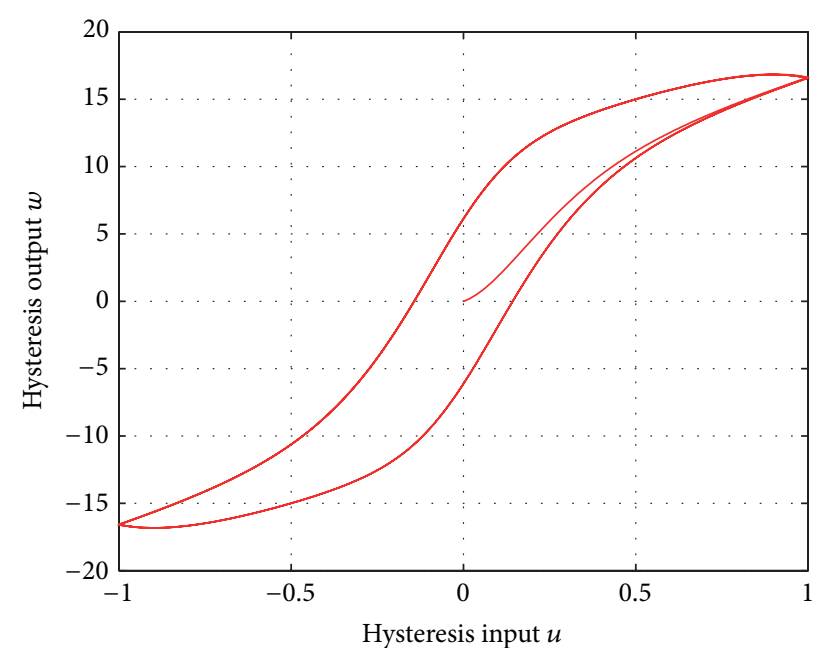

FIGURE 1: Hysteresis curves described by $\lambda(u)=10 \tanh 5 u+8 u$.

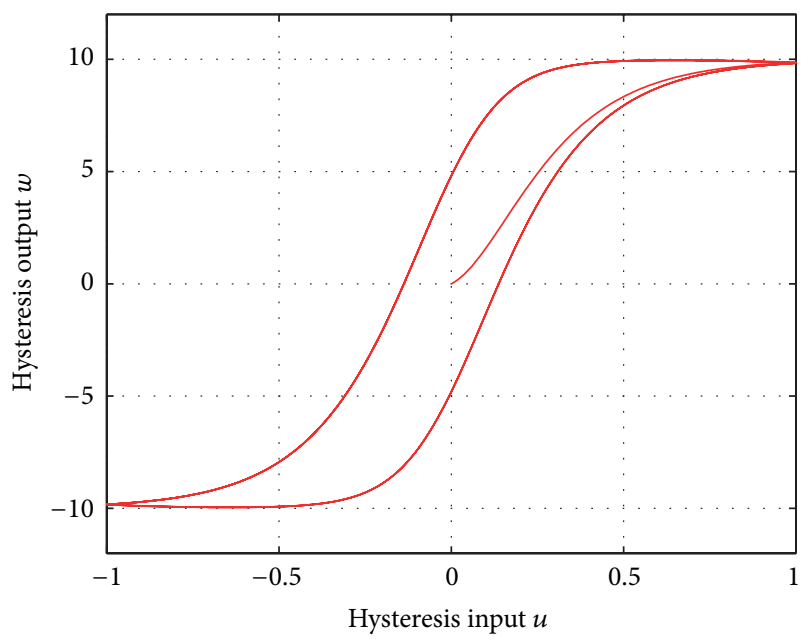

FIgURE 2: Hysteresis curves described by $\lambda(u)=10 \tanh 5 u$.

satisfying three properties; the described hysteresis curves are shown in Figures 1 and 2.

Under the previous three conditions, the Duhem model (3) can be solved explicitly for $u$ piecewise monotone as [14]

$$
w=\lambda(u)+\eta(u),
$$

where

$$
\begin{aligned}
\eta(u)= & {\left[w_{0}-\lambda\left(u_{0}\right)\right] e^{-\alpha\left(u-u_{0}\right) \operatorname{sgn}(\dot{u})} } \\
& +e^{-\alpha u \operatorname{sgn}(\dot{u})} \int_{u_{0}}^{u}[\psi(\zeta)-\dot{\lambda}(\zeta)] e^{\alpha \zeta \operatorname{sgn}(\dot{u})} d \zeta .
\end{aligned}
$$

For $\eta(u)$, if $w\left(u ; u_{0}, w_{0}\right)$ is the solution of (5) with initial values $\left(w_{0}, u_{0}\right)$, one has

$$
\begin{aligned}
& \lim _{u \rightarrow+\infty} \eta(u)=\lim _{u \rightarrow+\infty}\left[w\left(u ; u_{0}, w_{0}\right)-\lambda(u)\right]=0, \\
& \lim _{u \rightarrow-\infty} \eta(u)=\lim _{u \rightarrow-\infty}\left[w\left(u ; u_{0}, w_{0}\right)-\lambda(u)\right]=0 ;
\end{aligned}
$$

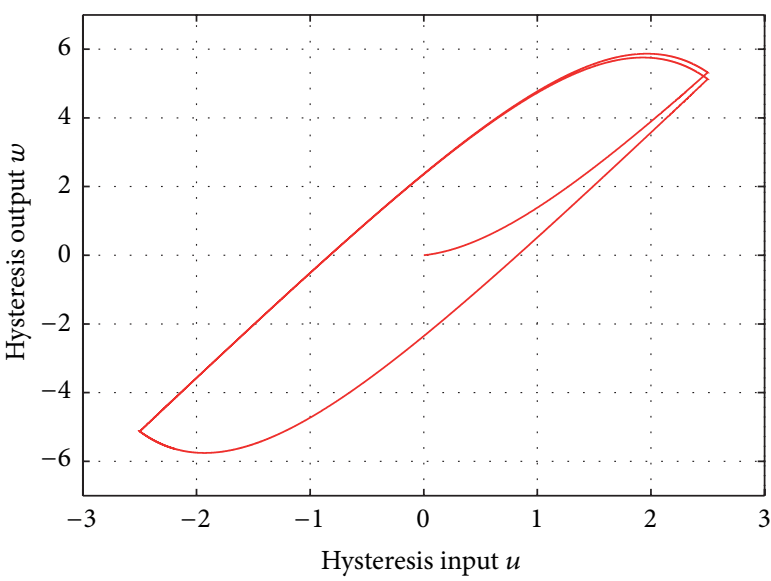

Figure 3: Hysteresis curves described by Backlash-like model.

then it can be deduced that $\eta(u)$ is bounded [14] easily. For simplicity, let $D$ denote the bound of $\eta(u)$, where $D$ is a positive constant.

Remark 6. When $f(v)=c u$ and $g(u)$ is a constant $B$, the Duhem model can be expressed as

$$
\frac{d w}{d t}=\alpha\left|\frac{d u}{d t}\right|[c u-w]+\frac{d u}{d t} B .
$$

When $c>B$, the Duhem model becomes the Backlashlike model defined in [9]. According to the above analysis, it is obvious that the Backlash-like model is a special case of the Duhem model. However, it should be noted that when $f(u)=c u$ and $g(u)=B$, Conditions 1 and 2 are not satisfied necessarily for the Duhem model. Similarly, (8) can be solved explicitly for the Backlash-like model:

$$
w=c u+\eta(u)
$$

with

$$
\begin{aligned}
\eta(u)= & {\left[w_{0}-c u_{0}\right] e^{-\alpha\left(u-u_{0}\right) \operatorname{sgn}(i)} } \\
& +e^{-\alpha u \operatorname{sgn}(i)} \int_{u_{0}}^{u}[B-c] e^{\alpha \zeta \operatorname{sgn}(\dot{u})} d \zeta .
\end{aligned}
$$

According to the analysis in [9], it has

$$
\begin{aligned}
& \lim _{u_{0} \rightarrow+\infty} \eta(u)=\lim _{u_{0} \rightarrow+\infty}\left[w\left(u ; u_{0}, w_{0}\right)-f(u)\right]=-\frac{c-B}{\alpha}, \\
& \lim _{u_{0} \rightarrow-\infty} \eta(u)=\lim _{u_{0} \rightarrow-\infty}\left[w\left(u ; u_{0}, w_{0}\right)-f(u)\right]=\frac{c-B}{\alpha} ;
\end{aligned}
$$

so the disturbance term $\varphi(v)$ is still bounded.

According to the previous proof, $D$ still can be used to denote the bound of $\eta(u)$ defined for Backlash-like model. As a comparison, when $\lambda(u)=c u=3.1635 u, \eta(u)=B=0.345$, and the input $u(t)=2.5 \sin (2.3 t)$, the Duhem model can be reexpressed as the Backlash-like model; then the curve of the Backlash-like model is shown in Figure 3. 


\section{Adaptive DSC Design and Stability Analysis}

In this section, the procedure for the design of adaptive dynamic surface controller and system stability will be given. Considering the characteristics of the hysteresis nonlinearities existing in the actual controlled plant, the following assumption is made for the hysteresis model (3).

Assumption 7. The function $\lambda(u)$ of Duhem hysteresis (3) is a smooth and strictly increasing function.

According to Condition 1 of Duhem model, $\lambda(0)=0$. Combining the derivative form of mean value theorem and Assumption 7 , there exists $\vartheta \in(\min (0, u), \max (0, u))$ such that

$$
\lambda(u)=\lambda(u)-\lambda(0)=\left.\frac{d \lambda(u)}{d u}\right|_{u=9}(u-0)=\dot{\lambda}(\vartheta) u .
$$

Then $w$ is rewritten as

$$
w=\dot{\lambda}(\vartheta) u+\eta(u)
$$

then the system (1) is expressed as

$$
\begin{gathered}
\dot{x}_{i}=\theta_{i} f_{i}\left(\bar{x}_{i}\right)+g_{i} x_{i+1}+d_{i}(x, t), \quad i=1, \ldots, n-1, \\
\dot{x}_{n}=\theta_{n} f_{n}(x)+g_{n}[\dot{\lambda}(\vartheta) u+\eta(u)]+d_{n}(x, t), \\
y=x_{1} .
\end{gathered}
$$

Since the sign of the control gain $g_{n} \dot{\lambda}(\vartheta)$ is unknown, one useful lemma is given as follows.

Lemma 8 (see [29]). Let $V(\cdot), \zeta(\cdot)$ be the smooth functions defined on $\left[0, t_{f}\right)$ with $V(t) \geq 0$, for all $t \in\left[0, t_{f}\right)$, and let $N(\cdot)$ be an ever smooth Nussbaum-type function. If the following inequalities hold

$$
V(t) \leq c_{0}+e^{-c_{1} t} \int_{0}^{t}[G(\cdot) N(\zeta)+1] \dot{\zeta} e^{c_{1} \tau} d \tau,
$$

where $c_{0}$ represents some suitable constant, $c_{1}$ is a positive constant, and $G(\cdot)$ is a time-varying parameter which takes values in the unknown closed intervals $I=\left[l^{-}, l^{+}\right]$, with $0 \notin$ $I$, then $V(t), \zeta(t)$, and $\int_{0}^{t} G(\cdot) N(\zeta) \dot{\zeta} d \tau$ must be bounded on $\left[0, t_{f}\right)$.

4.1. Adaptive DSC Design. Following the DSC procedure, the coordinate transformation is made as follows:

$$
z_{1}=x_{1}-y_{d}, \quad z_{i}=x_{i}-s_{i-1}, \quad i=2, \ldots, n,
$$

where $s_{i-1}$ are output of the filter (17).

The first-order low pass filters and the boundary filter errors $e_{i}$ are defined as

$$
\begin{gathered}
\tau_{i} \dot{s}_{i}+s_{i}=\alpha_{i}, \quad s_{i}(0)=\alpha_{i}(0), \\
e_{i}=s_{i}-\alpha_{i},
\end{gathered}
$$

where $\tau_{i}$ are the filter time constant and $\alpha_{i}$ are the filter input, which are also the virtual control law for the $i$ th subsystem specified hereinafter, $i=1, \ldots, n-1$.
Step 1. Considering the first equation in (14) and invoking (16)-(18), the time derivative of $z_{1}$ is given by

$$
\begin{aligned}
\dot{z}_{1} & =\theta_{1} f_{1}\left(\bar{x}_{1}\right)+g_{1} x_{2}+d_{1}(x, t)-\dot{y}_{d} \\
& =\theta_{1} f_{1}\left(\bar{x}_{1}\right)+g_{1}\left[z_{2}+\alpha_{1}+e_{1}\right]+d_{1}(x, t)-\dot{y}_{d} .
\end{aligned}
$$

Define the Lyapunov function candidate

$$
V_{1}=\frac{1}{2}\left(\frac{1}{g_{1}} z_{1}^{2}+\frac{1}{\gamma_{\theta_{1}}} \widetilde{\theta}_{g_{1}}^{2}+\frac{1}{\gamma_{b_{1}}} \widetilde{b}_{g_{1}}^{2}+\frac{1}{\gamma_{\bar{g}_{1}}} \widetilde{\bar{g}}_{g_{1}}^{2}\right),
$$

where $\widetilde{\theta}_{g_{1}}=\theta_{g_{1}}-\widehat{\theta}_{g_{1}}, \widetilde{b}_{g_{1}}=b_{g_{1}}-\widehat{b}_{g_{1}}$, and $\widetilde{\bar{g}}_{g_{1}}=\bar{g}_{g_{1}}-\widehat{\bar{g}}_{g_{1}}$ with $\widehat{\theta}_{g_{1}}, \widehat{b}_{g_{1}}$, and $\widehat{\bar{g}}_{g_{1}}$ as the estimates of $\theta_{g_{1}}=\theta_{1} / g_{1}, b_{g_{1}}=b_{1} / g_{1}$, and $\bar{g}_{g_{1}}=1 / g_{1}$, respectively. $\gamma_{\theta_{1}}, \gamma_{b_{1}}$, and $\gamma_{\bar{g}_{1}}$ are positive design parameters.

Note that the following inequalities hold [30]:

$$
\begin{aligned}
z_{i} d_{i}(x, t) & \leq b_{i}\left|z_{i}\right| \rho_{i}\left(\bar{x}_{i}\right) \\
& \leq b_{i} z_{i} \rho_{i}\left(\bar{x}_{i}\right) \tanh \left(\frac{z_{i} \rho_{i}\left(\bar{x}_{i}\right)}{\omega}\right)+0.2785 \omega b_{i},
\end{aligned}
$$

where $\omega>0, i=1, \ldots, n$.

Based on (19) and (21), it has

$$
\begin{aligned}
& \dot{V}_{1}=\frac{z_{1}}{g_{1}}\left(\theta_{1} f_{1}\left(\bar{x}_{1}\right)+g_{1}\left[z_{2}+\alpha_{1}+e_{1}\right]+d_{1}(x, t)-\dot{y}_{d}\right) \\
& +\frac{1}{\gamma_{\theta_{1}}} \widetilde{\theta}_{g_{1}} \dot{\tilde{\theta}}_{g_{1}}+\frac{1}{\gamma_{b_{1}}} \widetilde{b}_{g_{1}} \dot{\widetilde{b}}_{g_{1}}+\frac{1}{\gamma_{\bar{g}_{1}}} \widetilde{\bar{g}}_{g_{1}} \dot{\bar{g}}_{g_{1}} \\
& \leq z_{1}\left(\theta_{g_{1}} f_{1}\left(\bar{x}_{1}\right)+\alpha_{1}\right. \\
& \left.+b_{g_{1}} \rho_{1}\left(\bar{x}_{1}\right) \tanh \left(\frac{z_{1} \rho_{1}\left(\bar{x}_{1}\right)}{\omega}\right)-\bar{g}_{g_{1}} \dot{y}_{d}\right) \\
& +z_{1} z_{2}+z_{1} e_{1}+0.2785 \omega b_{g_{1}}+\frac{1}{\gamma_{\theta_{1}}} \widetilde{\theta}_{g_{1}} \dot{\tilde{\theta}}_{g_{1}} \\
& +\frac{1}{\gamma_{b_{1}}} \widetilde{b}_{g_{1}} \dot{\widetilde{b}}_{g_{1}}+\frac{1}{\gamma_{\bar{g}_{1}}} \widetilde{\bar{g}}_{g_{1}} \dot{\bar{g}}_{g_{1}} \\
& \leq z_{1}\left(\widehat{\theta}_{g_{1}} f_{1}\left(\bar{x}_{1}\right)+\alpha_{1}+\widehat{b}_{g_{1}} \rho_{1}\left(\bar{x}_{1}\right) \tanh \left(\frac{z_{1} \rho_{1}\left(\bar{x}_{1}\right)}{\omega}\right)\right. \\
& \left.-\widehat{\bar{g}}_{g_{1}} \dot{y}_{d}\right) \\
& +z_{1} z_{2}+z_{1} e_{1}+0.2785 \omega b_{g_{1}} \\
& +\tilde{\theta}_{g_{1}}\left(z_{1} f_{1}\left(\bar{x}_{1}\right)-\frac{1}{\gamma_{\theta_{1}}} \dot{\hat{\theta}}_{g_{1}}\right) \\
& +\widetilde{b}_{g_{1}}\left(z_{1} \rho_{1}\left(\bar{x}_{1}\right) \tanh \left(\frac{z_{1} \rho_{1}\left(\bar{x}_{1}\right)}{\omega}\right)-\frac{1}{\gamma_{b_{1}}} \dot{\hat{b}}_{g_{1}}\right) \\
& +\widetilde{\bar{g}}_{g_{1}}\left(-z_{1} \dot{y}_{d}-\frac{1}{\gamma_{\bar{g}_{1}}} \dot{\overline{\bar{g}}}_{g_{1}}\right) \text {. }
\end{aligned}
$$


The virtual control law $\alpha_{1}$ and the adaptive laws $\widehat{\theta}_{g_{1}}, \widehat{b}_{g_{1}}$, and $\hat{\bar{g}}_{g_{1}}$ are designed as

$$
\begin{gathered}
\alpha_{1}=-k_{1} z_{1}-\widehat{\theta}_{g_{1}} f_{1}\left(\bar{x}_{1}\right)-\widehat{b}_{g_{1}} \rho_{1}\left(\bar{x}_{1}\right) \tanh \left(\frac{z_{1} \rho_{1}\left(\bar{x}_{1}\right)}{\omega}\right) \\
+\hat{\bar{g}}_{g_{1}} \dot{y}_{d}, \\
\dot{\hat{\theta}}_{g_{1}}=\gamma_{\theta_{1}}\left(z_{1} f_{1}\left(\bar{x}_{1}\right)-\omega_{1} \widehat{\theta}_{g_{1}}\right), \\
\dot{\vec{b}}_{g_{1}}=\gamma_{b_{1}}\left(z_{1} \rho_{1}\left(\bar{x}_{1}\right) \tanh \left(\frac{z_{1} \rho_{1}\left(\bar{x}_{1}\right)}{\omega}\right)-\mu_{1} \widehat{b}_{g_{1}}\right), \\
\dot{\bar{g}}_{g_{1}}=\gamma_{\bar{g}_{1}}\left(-z_{1} \dot{y}_{d}-v_{1} \hat{\bar{g}}_{g_{1}}\right),
\end{gathered}
$$

where $k_{1}, \Phi_{1}, \mu_{1}$, and $\nu_{1}$ are positive design parameters.

Substituting (23) into (22), we obtain

$$
\begin{aligned}
\dot{V}_{1} \leq & -k_{1} z_{1}^{2}+z_{1} z_{2}+z_{1} e_{1}+0.2785 \omega b_{g_{1}}+\omega_{1} \widetilde{\theta}_{g_{1}} \widehat{\theta}_{g_{1}} \\
& +\mu_{1} \widetilde{b}_{g_{1}} \widehat{b}_{g_{1}}+v_{1} \widetilde{\bar{g}}_{g_{1}} \hat{\bar{g}}_{g_{1}} .
\end{aligned}
$$

By using the following inequalities

$$
\begin{aligned}
& \varpi_{1} \widetilde{\theta}_{g_{1}} \widehat{\theta}_{g_{1}} \leq \frac{\varpi_{1}}{2}\left(-\widetilde{\theta}_{g_{1}}^{2}+\theta_{g_{1}}^{2}\right), \\
& \mu_{1} \widetilde{b}_{g_{1}} \widehat{b}_{g_{1}} \leq \frac{\mu_{1}}{2}\left(-\widetilde{b}_{g_{1}}^{2}+b_{g_{1}}^{2}\right), \\
& v_{1} \widetilde{\bar{g}}_{g_{1}} \hat{\bar{g}}_{g_{1}} \leq \frac{v_{1}}{2}\left(-\widetilde{\bar{g}}_{g_{1}}^{2}+\bar{g}_{g_{1}}^{2}\right),
\end{aligned}
$$

we have

$$
\begin{aligned}
\dot{V}_{1} \leq & -k_{1} z_{1}^{2}-\frac{\omega_{1}}{2} \widetilde{\theta}_{g_{1}}^{2}-\frac{\mu_{1}}{2} \widetilde{b}_{g_{1}}^{2}-\frac{\nu_{1}}{2} \widetilde{\bar{g}}_{g_{1}}^{2}+z_{1} z_{2}+z_{1} e_{1} \\
& +0.2785 \omega b_{g_{1}}+\frac{\omega_{1}}{2} \theta_{g_{1}}^{2}+\frac{\mu_{1}}{2} b_{g_{1}}^{2}+\frac{\nu_{1}}{2} \bar{g}_{g_{1}}^{2} .
\end{aligned}
$$

Step $i(2 \leq i \leq n-1)$. Considering (17) and (18), and $z_{i}=$ $x_{i}-s_{i-1}$, it has

$$
\begin{gathered}
s_{i}=e_{i}+\alpha_{i}, \quad \dot{s}_{i}=-\frac{e_{i}}{\tau_{i}}, \quad i=1, \ldots, n-1, \\
\dot{z}_{i}=\theta_{i} f_{i}\left(\bar{x}_{i}\right)+g_{i} x_{i+1}+d_{i}(x, t)+\frac{e_{i-1}}{\tau_{i-1}} \\
=\theta_{i} f_{i}\left(\bar{x}_{i}\right)+g_{i}\left[z_{i+1}+\alpha_{i}+e_{i}\right]+d_{i}(x, t)+\frac{e_{i-1}}{\tau_{i-1}} .
\end{gathered}
$$

Define the Lyapunov function candidate

$$
V_{i}=V_{i-1}+\frac{1}{2}\left(\frac{1}{g_{i}} z_{i}^{2}+\frac{1}{\gamma_{\theta_{i}}} \widetilde{\theta}_{g_{i}}^{2}+\frac{1}{\gamma_{b_{i}}} \widetilde{b}_{g_{i}}^{2}+\frac{1}{\gamma_{\bar{g}_{i}}} \widetilde{\bar{g}}_{g_{i}}^{2}\right),
$$

where $\widetilde{\theta}_{g_{i}}=\theta_{g_{i}}-\widehat{\theta}_{g_{i}}, \widetilde{b}_{g_{i}}=b_{g_{i}}-\widehat{b}_{g_{i}}$, and $\widetilde{\bar{g}}_{g_{i}}=\bar{g}_{g_{i}}-\widehat{\bar{g}}_{g_{i}}$ with $\widehat{\theta}_{g_{i}}, \widehat{b}_{g_{i}}$, and $\hat{\bar{g}}_{g_{i}}$ as the estimates of $\theta_{g_{i}}=\theta_{i} / g_{i}, b_{g_{i}}=b_{i} / g_{i}$, and $\bar{g}_{g_{i}}=1 / g_{i}$, respectively. $\gamma_{\theta_{i}}, \gamma_{b_{i}}$, and $\gamma_{\bar{g}_{i}}$ are positive design parameters. by

Based on (21) and (28), the time derivative of $V_{i}$ is given

$$
\begin{aligned}
& \dot{V}_{i}=\dot{V}_{i-1}+\frac{z_{i}}{g_{i}}\left(\theta_{i} f_{i}\left(\bar{x}_{i}\right)+g_{i}\left[z_{i+1}+\alpha_{i}+e_{i}\right]\right. \\
& \left.+d_{i}(x, t)+\frac{e_{i-1}}{\tau_{i-1}}\right) \\
& +\frac{1}{\gamma_{\theta_{i}}} \widetilde{\theta}_{g_{i}} \dot{\tilde{\theta}}_{g_{i}}+\frac{1}{\gamma_{b_{i}}} \widetilde{b}_{g_{i}} \dot{\widetilde{b}}_{g_{i}}+\frac{1}{\gamma_{\bar{g}_{i}}} \widetilde{\bar{g}}_{g_{i}} \dot{\overline{\bar{g}}}_{g_{i}} \\
& \leq \dot{V}_{i-1}+z_{i}\left(z_{i-1}+\theta_{g_{i}} f_{i}\left(\bar{x}_{i}\right)+\alpha_{i}\right. \\
& \left.+b_{g_{i}} \rho_{i}\left(\bar{x}_{i}\right) \tanh \left(\frac{z_{i} \rho_{i}\left(\bar{x}_{i}\right)}{\omega}\right)+\bar{g}_{g_{i}} \frac{e_{i-1}}{\tau_{i-1}}\right) \\
& +z_{i} z_{i+1}-z_{i} z_{i-1}+z_{i} e_{i}+0.2785 \omega b_{g_{i}} \\
& +\frac{1}{\gamma_{\theta_{i}}} \widetilde{\theta}_{g_{i}} \dot{\tilde{\theta}}_{g_{i}}+\frac{1}{\gamma_{b_{i}}} \widetilde{b}_{g_{i}} \dot{\widetilde{b}}_{g_{i}}+\frac{1}{\gamma_{\bar{g}_{i}}} \widetilde{\bar{g}}_{g_{i}} \dot{\bar{g}}_{g_{i}} \\
& \leq \dot{V}_{i-1}+z_{i}\left(z_{i-1}+\hat{\theta}_{g_{i}} f_{i}\left(\bar{x}_{i}\right)+\alpha_{i}\right. \\
& \left.+\widehat{b}_{g_{i}} \rho_{i}\left(\bar{x}_{i}\right) \tanh \left(\frac{z_{i} \rho_{i}\left(\bar{x}_{i}\right)}{\omega}\right)+\hat{\bar{g}}_{g_{i}} \frac{e_{i-1}}{\tau_{i-1}}\right) \\
& +z_{i} z_{i+1}-z_{i} z_{i-1}+z_{i} e_{i}+0.2785 \omega b_{g_{i}} \\
& +\widetilde{\theta}_{g_{i}}\left(z_{i} f_{i}\left(\bar{x}_{i}\right)-\frac{1}{\gamma_{\theta_{i}}} \dot{\hat{\theta}}_{g_{i}}\right) \\
& +\widetilde{b}_{g_{i}}\left(z_{i} \rho_{i}\left(\bar{x}_{i}\right) \tanh \left(\frac{z_{i} \rho_{i}\left(\bar{x}_{i}\right)}{\omega}\right)-\frac{1}{\gamma_{b_{i}}} \dot{\vec{b}}_{g_{i}}\right) \\
& +\widetilde{\bar{g}}_{g_{i}}\left(z_{i} \frac{e_{i-1}}{\tau_{i-1}}-\frac{1}{\gamma_{\bar{g}_{i}}} \dot{\overline{\bar{g}}}_{g_{i}}\right) .
\end{aligned}
$$

The virtual control law $\alpha_{i}$ and the adaptive update laws $\widehat{\theta}_{g_{i}}, \widehat{b}_{g_{i}}$, and $\widehat{\bar{g}}_{g_{i}}$ are designed as

$$
\begin{gathered}
\alpha_{i}=-k_{i} z_{i}-z_{i-1}-\widehat{\theta}_{g_{i}} f_{i}\left(\bar{x}_{i}\right)-\widehat{b}_{g_{i}} \rho_{i}\left(\bar{x}_{i}\right) \tanh \left(\frac{z_{i} \rho_{i}\left(\bar{x}_{i}\right)}{\omega}\right) \\
-\hat{\bar{g}}_{g_{i}} \frac{e_{i-1}}{\tau_{i-1}} \\
\dot{\hat{b}}_{g_{i}}=\gamma_{b_{i}}\left(z_{i} \rho_{i}\left(\bar{x}_{i}\right) \tanh \left(\frac{z_{i} \rho_{i}\left(\bar{x}_{i}\right)}{\omega}\right)-\mu_{i} \widehat{b}_{g_{i}}\right) \\
\dot{\overline{\bar{g}}}_{g_{i}}=\gamma_{\bar{g}_{i}}\left(z_{i} \frac{e_{i-1}}{\tau_{i-1}}-v_{i} \hat{\bar{g}}_{g_{i}}\right)
\end{gathered}
$$

where $k_{i}, \omega_{i}, \mu_{i}$, and $v_{i}$ are positive design parameters. 
Considering the following inequalities

$$
\begin{aligned}
& \omega_{i} \widetilde{\theta}_{g_{i}} \widehat{\theta}_{g_{i}} \leq \frac{\omega_{i}}{2}\left(-\widetilde{\theta}_{g_{i}}^{2}+\theta_{g_{i}}^{2}\right), \\
& \mu_{i} \widetilde{b}_{g_{i}} \widehat{b}_{g_{i}} \leq \frac{\mu_{i}}{2}\left(-\widetilde{b}_{g_{i}}^{2}+b_{g_{i}}^{2}\right), \\
& v_{i} \widetilde{\bar{g}}_{g_{i}} \widehat{\bar{g}}_{g_{i}} \leq \frac{\nu_{i}}{2}\left(-\widetilde{\bar{g}}_{g_{i}}^{2}+\bar{g}_{g_{i}}^{2}\right),
\end{aligned}
$$

we have

$$
\begin{gathered}
\dot{V}_{i} \leq-\sum_{j=1}^{i} k_{j} z_{j}^{2}-\sum_{j=1}^{i}\left(\frac{\omega_{1}}{2} \widetilde{\theta}_{g_{1}}^{2}+\frac{\mu_{1}}{2} \widetilde{b}_{g_{1}}^{2}+\frac{\nu_{i}}{2} \widetilde{\bar{g}}_{g_{1}}^{2}\right) \\
+z_{i} z_{i+1}+\sum_{j=1}^{i}\left(z_{j} e_{j}+0.2785 \omega b_{g_{j}}+\frac{\omega_{j}}{2} \theta_{g_{j}}^{2}\right. \\
\left.+\frac{\mu_{i}}{2} b_{g_{j}}^{2}+\frac{\nu_{i}}{2} \bar{g}_{g_{j}}^{2}\right) .
\end{gathered}
$$

Step $n$. The actual control law $u$ will be designed in this step. Considering $z_{n}=x_{n}-s_{n-1}$ and $\dot{s}_{n-1}=-e_{n-1} / \tau_{n-1}$, the time derivative of $z_{n}$ is given by

$$
\dot{z}_{n}=\theta_{n} f_{n}(x)+g_{n}[\dot{\lambda}(\vartheta) u+\eta(u)]+d_{n}(x, t)+\frac{e_{n-1}}{\tau_{n-1}} .
$$

Define the Lyapunov function candidate

$$
V_{n}=V_{n-1}+\frac{1}{2}\left(z_{n}^{2}+\frac{1}{\gamma_{\theta_{n}}} \widetilde{\theta}_{g_{n}}^{2}+\frac{1}{\gamma_{b_{n}}} \widetilde{b}_{g_{n}}^{2}+\frac{1}{\gamma_{\bar{g}_{n}}} \widetilde{\bar{g}}_{g_{n}}^{2}\right),
$$

where $\widetilde{\theta}_{g_{n}}=\theta_{g_{n}}-\widehat{\theta}_{g_{n}}, \widetilde{b}_{g_{n}}=b_{g_{n}}-\widehat{b}_{g_{n}}$, and $\widetilde{\bar{g}}_{g_{n}}=\bar{g}_{g_{n}}-\widehat{\bar{g}}_{g_{n}}$ with $\widehat{\theta}_{g_{n}}, \widehat{b}_{g_{n}}$, and $\widehat{\bar{g}}_{g_{n}}$ as the estimates of $\theta_{g_{n}}=\theta_{n}, b_{g_{n}}=b_{n}$, and $\bar{g}_{g_{n}}=g_{n} D$, respectively. $\gamma_{\theta_{n}}, \gamma_{b_{n}}$, and $\gamma_{\bar{g}_{n}}$ are positive design parameters.

Based on (37), we have

$$
\begin{aligned}
\dot{V}_{n}= & \dot{V}_{n-1}+z_{n}\left(\theta_{n} f_{n}(x)+g_{n}[\dot{\lambda}(\vartheta) u+\eta(u)]\right. \\
& \left.+d_{n}(x, t)+\frac{e_{n-1}}{\tau_{n-1}}\right) \\
& +\frac{1}{\gamma_{\theta_{i}}} \widetilde{\theta}_{g_{n}} \dot{\tilde{\theta}}_{g_{n}}+\frac{1}{\gamma_{b_{n}}} \widetilde{b}_{g_{n}} \dot{\widetilde{b}}_{g_{n}}+\frac{1}{\gamma_{\bar{g}_{n}}} \widetilde{\bar{g}}_{g_{n}} \dot{\tilde{\bar{g}}}_{g_{n}} \\
= & \dot{V}_{n-1}+z_{n} \theta_{n} f_{n}(x)+z_{n} g_{n} \dot{\lambda}(\vartheta) u \\
& +z_{n} g_{n} \eta(u)+z_{n} d_{n}(x, t) \\
& +z_{n} \frac{e_{n-1}}{\tau_{n-1}}+\frac{1}{\gamma_{\theta_{n}}} \widetilde{\theta}_{g_{n}} \dot{\tilde{\theta}}_{g_{n}}+\frac{1}{\gamma_{b_{n}}} \widetilde{b}_{g_{n}} \dot{\widetilde{b}}_{g_{n}}+\frac{1}{\gamma_{\bar{g}_{n}}} \widetilde{\bar{g}}_{g_{n}} \dot{\overline{\bar{g}}}_{g_{n}} .
\end{aligned}
$$

Similar to (21), the following inequalities are used:

$$
z_{n} g_{n} \leq \bar{g}_{g_{n}}\left|z_{n}\right| \leq \bar{g}_{g_{n}} z_{n} \tanh \left(\frac{z_{n}}{\omega}\right)+0.2785 \omega \bar{g}_{g_{n}} ;
$$

we have

$$
\begin{aligned}
\dot{V}_{n} \leq & \dot{V}_{n-1}+z_{n} \theta_{n} f_{n}(x)+z_{n} g_{n} \dot{\lambda}(\vartheta) u \\
& +\bar{g}_{g_{n}} z_{n} \tanh \left(\frac{z_{n}}{\omega}\right)+0.2785 \omega \bar{g}_{g_{n}}+0.2785 \omega b_{n} \\
& +b_{n} z_{n} \rho_{n}(x) \tanh \left(\frac{z_{n} \rho_{n}(x)}{\omega}\right)+z_{n} \frac{e_{n-1}}{\tau_{n-1}} \\
& +\frac{1}{\gamma_{\theta_{n}}} \tilde{\theta}_{g_{n}} \dot{\tilde{\theta}}_{g_{n}}+\frac{1}{\gamma_{b_{n}}} \tilde{b}_{g_{n}} \dot{\tilde{b}}_{g_{n}}+\frac{1}{\gamma_{\bar{g}_{n}}} \tilde{\bar{g}}_{g_{n}} \dot{\bar{g}}_{g_{n}} \\
\leq & \dot{V}_{n-1}+z_{n} z_{n-1}+z_{n} \widehat{\theta}_{n} f_{n}(x)+z_{n} g_{n} \dot{\lambda}(\vartheta) u \\
& +\hat{\bar{g}}_{z_{n}} \tanh \left(\frac{z_{n}}{\omega}\right) \\
& +0.2785 \omega \bar{g}_{g_{n}}+0.2785 \omega b_{n} \\
& +\widehat{b}_{n} z_{n} \rho_{n}(x) \tanh \left(\frac{z_{n} \rho_{n}(x)}{\omega}\right)+z_{n} \frac{e_{n-1}}{\tau_{n-1}} \\
& +\widetilde{\theta}_{g_{n}}\left(z_{n} f_{n}(x)-\frac{1}{\gamma_{\theta_{n}}} \dot{\hat{\theta}}_{g_{n}}\right) \\
& +\tilde{b}_{g_{n}}\left(z_{n} \rho_{n}(x) \tanh \left(\frac{z_{n} \rho_{n}(x)}{\omega}\right)-\frac{1}{\gamma_{b_{n}}} \dot{\tilde{b}}_{g_{n}}\right) \\
& \tilde{\bar{g}}_{g_{n}}\left(z_{n} \tanh \left(\frac{z_{n}}{\omega}\right)-\frac{1}{\gamma_{\bar{g}_{n}}}\right)-z_{n} z_{n-1} \cdot
\end{aligned}
$$

The actual control law $u$ and the adaptive laws $\zeta, \widehat{\theta}_{g_{n}}, \widehat{b}_{g_{n}}$, and $\widehat{\bar{g}}_{g_{n}}$ are designed as

$$
\begin{gathered}
u=N(\zeta)\left[k_{n} z_{n}+z_{n-1}+\widehat{\theta}_{g_{n}} f_{n}(x)+\hat{\bar{g}}_{g_{n}} \tanh \left(\frac{z_{n}}{\omega}\right)\right. \\
\left.+\widehat{b}_{g_{n}} \rho_{n}(x) \tanh \left(\frac{z_{n} \rho_{n}(x)}{\omega}\right)+\frac{e_{n-1}}{\tau_{n-1}}\right], \\
\dot{\zeta}=k_{n} z_{n}^{2}+z_{n} z_{n-1}+z_{n} \widehat{\theta}_{g_{n}} f_{n}(x)+\widehat{\bar{g}}_{g_{n}} z_{n} \tanh \left(\frac{z_{n}}{\omega}\right) \\
+z_{n} \widehat{b}_{g_{n}} \rho_{n}(x) \tanh \left(\frac{z_{n} \rho_{n}(x)}{\omega}\right)+z_{n} \frac{e_{n-1}}{\tau_{n-1}}, \\
\dot{\hat{\theta}}_{g_{n}}=\gamma_{\theta_{n}}\left(z_{n} f_{n}(x)-\omega_{n} \hat{\theta}_{g_{n}}\right), \\
\dot{\hat{b}}_{g_{n}}=\gamma_{b_{n}}\left(z_{n} \rho_{n}(x) \tanh \left(\frac{z_{n} \rho_{n}(x)}{\omega}\right)-\mu_{n} \widehat{b}_{g_{n}}\right), \\
\dot{\bar{g}}_{g_{n}}=\gamma_{\bar{g}_{n}}\left(z_{n} \tanh \left(\frac{z_{n}}{\omega}\right)-v_{n} \hat{\bar{g}}_{g_{n}}\right),
\end{gathered}
$$

where $k_{n}, \omega_{n}, \mu_{n}$, and $\nu_{n}$ are positive design parameters. Similarly, the following inequalities will be utilized:

$$
\begin{aligned}
& \omega_{n} \widetilde{\theta}_{g_{n}} \widehat{\theta}_{g_{n}} \leq \frac{\omega_{n}}{2}\left(-\widetilde{\theta}_{g_{n}}^{2}+\theta_{g_{n}}^{2}\right), \\
& \mu_{n} \widetilde{b}_{g_{n}} \widehat{b}_{g_{n}} \leq \frac{\mu_{n}}{2}\left(-\widetilde{b}_{g_{n}}^{2}+b_{g_{n}}^{2}\right), \\
& v_{n} \widetilde{\bar{g}}_{g_{n}} \widehat{\bar{g}}_{g_{n}} \leq \frac{v_{n}}{2}\left(-\widetilde{\bar{g}}_{g_{n}}^{2}+\bar{g}_{g_{n}}^{2}\right) ;
\end{aligned}
$$


then, we obtain

$$
\begin{gathered}
\dot{V}_{n} \leq-\sum_{j=1}^{n} k_{j} z_{j}^{2}-\sum_{j=1}^{n}\left(\frac{\omega_{j}}{2} \widetilde{\theta}_{g_{j}}^{2}+\frac{\mu_{j}}{2} \widetilde{b}_{g_{j}}^{2}+\frac{v_{j}}{2} \widetilde{\bar{g}}_{g_{j}}^{2}\right) \\
+\left[g_{n} \dot{\lambda}(\vartheta) N(\zeta)+1\right] \dot{\zeta}+0.2785 \omega \bar{g}_{g_{n}} \\
+\sum_{j=1}^{n-1}\left(z_{j} e_{j}\right)+\sum_{j=1}^{n}\left(0.2785 \omega b_{g_{j}}+\frac{\omega_{j}}{2} \theta_{g_{j}}^{2}+\frac{\mu_{i}}{2} b_{g_{j}}^{2}\right. \\
\left.+\frac{v_{i}}{2} \bar{g}_{g_{j}}^{2}\right) .
\end{gathered}
$$

4.2. Stability Analysis. In this subsection, the uniform ultimate boundedness of all signals in the closed-loop system will be proven.

From (27) and (31), we have

$$
\begin{aligned}
& \dot{e}_{i}=\dot{s}_{i}-\dot{\alpha}_{i} \\
& =-\frac{e_{i}}{\tau_{i}}+\left(\frac{\partial \alpha_{i}}{\partial z_{i}} \dot{z}_{i}+\frac{\partial \alpha_{i}}{\partial \hat{\theta}_{g_{i}}} \dot{\hat{\theta}}_{g_{i}}+\frac{\partial \alpha_{i}}{\partial \widehat{\hat{b}}_{g_{i}}} \dot{\vec{b}}_{g_{i}}+\frac{\partial \alpha_{i}}{\partial \hat{\bar{g}}_{g_{i}}} \dot{\overline{\bar{g}}}_{g_{i}}\right) \\
& =-\frac{e_{i}}{\tau_{i}}+B_{i}\left(z_{1}, \ldots, z_{i}, e_{1}, \ldots, e_{i-1}, \widehat{\theta}_{g_{1}}, \ldots, \widehat{\theta}_{g_{i}},\right. \\
& \left.\widehat{b}_{g_{1}}, \ldots, \widehat{b}_{g_{i}}, \widehat{\bar{g}}_{g_{1}}, \ldots, \widehat{g}_{g_{i}}, y_{d}, \dot{y}_{d}, \ddot{y}_{d}\right),
\end{aligned}
$$

where $\quad B_{i}\left(z_{1}, \ldots, z_{i}, e_{1}, \ldots, e_{i-1}, \widehat{\theta}_{g_{1}}, \ldots, \widehat{\theta}_{g_{i}}, \widehat{b}_{g_{1}}, \ldots, \widehat{b}_{g_{i}}, \widehat{\bar{g}}_{g_{1}}\right.$, $\left.\ldots, \widehat{g}_{g_{i}}, y_{d}, \dot{y}_{d}, \ddot{y}_{d}\right)=\left(\partial \alpha_{i} / \partial z_{i}\right) \dot{z}_{i}+\left(\partial \alpha_{i} / \partial \widehat{\theta}_{g_{i}}\right) \dot{\hat{\theta}}_{g_{i}}+$ $\left(\partial \alpha_{i} / \partial \widehat{b}_{g_{i}}\right) \dot{\vec{b}}_{g_{i}}+\left(\partial \alpha_{i} / \partial \widehat{\bar{g}}_{g_{i}}\right) \dot{\bar{g}}_{g_{i}}$ are the continuous functions, $i=1, \ldots, n-1$.

To establish the boundedness of the closed-loop system, the following Lyapunov function candidate is defined as

$$
V=V_{n}+\frac{1}{2} \sum_{i=1}^{n-1} e_{i}^{2} .
$$

The main results can be summarized as follows.

Theorem 9. Consider the closed-loop system consisting of the plant (1), the controller (42), and adaptation laws (43) under Assumptions 1-7. If $V(0) \leq P_{0}$ for any $P_{0}>0$, there exist the appropriate design parameters $k_{i}, \tau_{i}, \gamma_{\theta_{i}}, \gamma_{b_{i}}, \gamma_{\bar{g}_{i}}, \omega_{i}, \mu_{i}$, $v_{i}$, and $\omega$, such that all signals in the closed-loop system are semiglobally uniformly ultimately bounded.

Proof. Define the set $\Omega_{i}:=\left\{\left[z_{1}, \ldots, z_{i}, e_{1}, \ldots, e_{i-1}, \widehat{\theta}_{g_{1}}, \ldots\right.\right.$, $\left.\left.\widehat{\theta}_{g_{i}}, \widehat{b}_{g_{1}}, \ldots, \widehat{b}_{g_{i}}, \widehat{\bar{g}}_{g_{1}}, \ldots, \widehat{g}_{g_{i}}\right]: V_{i}+\sum_{j=1}^{i-1} e_{j}^{2} \leq 2 P_{0}\right\} \subset R^{5 i-1}$. From Assumption 1 and $V(0) \leq P_{0}$ for any $P_{0}>0$, the set $\Omega_{d}$ and $\Omega_{i}$ are compact in $R^{3}$ and $R^{5 i-1}$. Thus,
$B_{i}\left(z_{1}, \ldots, z_{i}, e_{1}, \ldots, e_{i-1}, \widehat{\theta}_{g_{1}}, \ldots, \widehat{\theta}_{g_{i}}, \widehat{b}_{g_{1}}, \ldots, \widehat{b}_{g_{i}}, \widehat{\bar{g}}_{g_{1}}, \ldots, \widehat{g}_{g_{i}}\right.$, $y_{d}, \dot{y}_{d}, \ddot{y}_{d}$ ) have a maximum value $M_{i}, i=1, \ldots, n-1$, on $\Omega_{d} \times \Omega_{i}$. Equation (46) can be further derived as

$$
\begin{gathered}
e_{i} \dot{e} \leq-\frac{e_{i}^{2}}{\tau_{i}}+\mid e_{i} B_{i}\left(z_{1}, \ldots, z_{i}, e_{1}, \ldots, e_{i-1}, \widehat{\theta}_{g_{1}}, \ldots, \widehat{\theta}_{g_{i}},\right. \\
\left.\widehat{b}_{g_{1}}, \ldots, \widehat{b}_{g_{i}}, \widehat{\bar{g}}_{g_{1}}, \ldots, \widehat{g}_{g_{i}}, y_{d}, \dot{y}_{d}, \ddot{y}_{d}\right) \mid \\
\leq-\frac{e_{i}^{2}}{\tau_{i}}+\frac{1}{2} e_{i}^{2}+\frac{1}{2} M_{i}^{2} .
\end{gathered}
$$

The derivative of $V$ with respect to $t$ follows from (45), (46), and (48) that

$$
\begin{aligned}
\dot{V} \leq & -\sum_{j=1}^{n} k_{j} z_{j}^{2}-\sum_{j=1}^{n}\left(\frac{\Phi_{j}}{2} \widetilde{\theta}_{g_{j}}^{2}+\frac{\mu_{j}}{2} \widetilde{b}_{g_{j}}^{2}+\frac{\nu_{j}}{2} \widetilde{\bar{g}}_{g_{j}}^{2}\right) \\
& +\left[g_{n} \dot{\lambda}(\vartheta) N(\zeta)+1\right] \dot{\zeta}+0.2785 \omega \bar{g}_{g_{n}}+\sum_{j=1}^{n-1}\left(z_{j} e_{j}\right) \\
& +\sum_{j=1}^{n}\left(0.2785 \omega b_{g_{j}}+\frac{\omega_{j}}{2} \theta_{g_{j}}^{2}+\frac{\mu_{i}}{2} b_{g_{j}}^{2}+\frac{\nu_{i}}{2} \bar{g}_{g_{j}}^{2}\right)+\sum_{i=1}^{n-1} e_{i} \dot{e}_{i} \\
\leq & -\sum_{j=1}^{n} k_{j} z_{j}^{2}-\sum_{j=1}^{n}\left(\frac{\omega_{j}}{2} \widetilde{\theta}_{g_{j}}^{2}+\frac{\mu_{j}}{2} \widetilde{b}_{g_{j}}^{2}+\frac{\nu_{j}}{2} \widetilde{\bar{g}}_{g_{j}}^{2}\right) \\
& +\left[g_{n} \dot{\lambda}(\vartheta) N(\zeta)+1\right] \dot{\zeta}+0.2785 \omega \bar{g}_{g_{n}}+\frac{1}{2} \sum_{j=1}^{n-1} z_{j}^{2} \\
& +\sum_{j=1}^{n}\left(0.2785 \omega b_{g_{j}}+\frac{\omega_{j}}{2} \theta_{g_{j}}^{2}+\frac{\mu_{i}}{2} b_{g_{j}}^{2}+\frac{\nu_{i}}{2} \bar{g}_{g_{j}}^{2}\right) \\
\leq & -\xi \xi V+\left[\sum_{j=1}^{n-1}\left(\frac{1}{\tau_{i}}-\frac{3}{2}\right) e_{j}^{2}+\sum_{i=1}^{n-1} \frac{1}{2} M_{i}^{2}\right. \\
& -\dot{\lambda}(\vartheta) N(\zeta)+1] \dot{\zeta}+\kappa,
\end{aligned}
$$

where

$$
\begin{aligned}
& \xi=\min \left\{2 g_{j}\left(k_{j}-\frac{1}{2}\right), 2 g_{n} k_{n}, \omega_{j} \gamma_{\theta_{j}}, \omega_{n} \gamma_{\theta_{n}},\right. j=1, \ldots, n-1, \\
&\left.\kappa=0.2785 \omega \bar{g}_{g_{n}} \gamma_{b_{j}}, \mu_{n} \gamma_{b_{n}}, \ldots, \nu_{j} \gamma_{\bar{g}_{j}}, \nu_{n} \gamma_{\bar{g}_{n}}, \frac{1}{\tau_{i}}-\frac{3}{2}\right\}, \\
& \quad+\sum_{j=1}^{n}\left(0.2785 \omega b_{g_{j}}+\frac{\omega_{j}}{2} \theta_{g_{j}}^{2}+\frac{\mu_{i}}{2} b_{g_{j}}^{2}+\frac{\nu_{i}}{2} \bar{g}_{g_{j}}^{2}\right) \\
&+\sum_{i=1}^{n-1} \frac{1}{2} M_{i}^{2} .
\end{aligned}
$$


Multiplying both sides of (49) by $e^{\xi t}$ yields

$$
\frac{d}{d t}\left(V e^{\xi t}\right) \leq \kappa e^{\xi t}+\left[g_{n} \dot{\lambda}(\vartheta) N(\zeta)+1\right] \dot{\zeta} e^{\xi t}
$$

Integrating $(51)$ over $[0, t]$, it is deduced that

$$
\begin{aligned}
V \leq & \frac{\kappa}{\xi}+\left[V(0)-\frac{\kappa}{\xi}\right] e^{\xi t} \\
& +e^{-\xi t} \int_{0}^{t}\left[g_{n} \dot{\lambda}(\vartheta) N(\zeta)+1\right] \dot{\zeta} e^{\xi \varrho} d \varrho \\
\leq & \frac{\kappa}{\xi}+V(0)+e^{-\xi t} \int_{0}^{t}\left[g_{n} \dot{\lambda}(\vartheta) N(\zeta)+1\right] \dot{\zeta} e^{\xi \varrho} d \varrho,
\end{aligned}
$$

where $c_{1}=(\kappa / \xi)+V(0)$.

From Condition 1 of Duhem model and Assumption 7, it is easily concluded that $\dot{\lambda}(u)>0$, and $\dot{\lambda}(u)$ is a bounded smooth even function of $u$, and hence $g_{n} \dot{\lambda}(\vartheta)$ is a nonzero bounded time-varying function. By using Lemma 8 , it implies that $V(t), \zeta, \int_{0}^{t}\left[g_{n} \dot{\lambda}(\vartheta) N(\zeta)+1\right] \dot{\zeta} e^{\xi \varrho} d \varrho$ are all bounded on $\left[0, t_{f}\right)$. From proposition 2 in [31], $t_{f}=\infty$, it can be concluded that all error signals $z_{1}, \ldots, z_{n}, \widetilde{\theta}_{g_{1}}, \ldots, \widetilde{\theta}_{g_{n}}$, $\widetilde{b}_{g_{1}}, \ldots, \widetilde{b}_{g_{n}}, \widetilde{\bar{g}}_{g_{1}}, \ldots, \widetilde{\bar{g}}_{g_{n}}, e_{i}, \ldots, e_{n-1}$ in the closed-loop system are semiglobally uniformly ultimately bounded.

\section{Numerical Example}

To demonstrate the effectiveness of the proposed control algorithm, in this section, one second-order nonlinear system with unknown Duhem hysteresis is considered:

$$
\begin{gathered}
\dot{x}_{1}=\theta_{1} f_{1}\left(\bar{x}_{1}\right)+g_{1} x_{2}+d_{1}(x, t), \\
\dot{x}_{2}=\theta_{2} f_{2}(x)+g_{2} w(u)+d_{2}(x, t), \\
y=x_{1},
\end{gathered}
$$

where $\theta_{1}, \theta_{2}, g_{1}$, and $g_{2}$ are unknown parameters, $d_{1}(x, t)$ and $d_{2}(x, t)$ are unknown disturbances, and $w$ is the output of the unknown hysteresis described by the Duhem model as (3). In the simulation, $\theta_{1}=0.1, \theta_{2}=1, g_{1}=1, g_{2}=1, f_{1}\left(x_{1}\right)=x_{1}^{2}$, $f_{2}(x)=-2 x_{1}-x_{2}, d_{1}(x, t)=0.2 \sin \left(x_{2}\right) \sin (t)$, and $d_{2}(x, t)=$ $0.1\left(x_{1}^{2}+x_{2}^{2}\right) \sin (t)$. Correspondingly, $b_{1}=0.2, \rho_{1}\left(x_{1}\right)=1$, $b_{2}=0.1$, and $\rho_{2}(x)=x_{1}^{2}+x_{2}^{2}$. For the Duhem model, $\alpha=1$, $\lambda(u)=5 \tanh 1.3 u+0.25 u$, and $\psi(u)=\dot{\lambda}(u)\left(1-e^{(-2.3|u|)}\right)$. The objective is to make the output $y$ of system (53) to track the desired trajectory $y_{d}=\sin (1.5 t)-0.3 \cos (t)$.

In this simulation, the initial values of adaptive laws are selected as $\widehat{\theta}_{g_{1}}=0, \widehat{\theta}_{g_{2}}=0, \widehat{b}_{g_{1}}=0, \widehat{b}_{g_{2}}=0, \widehat{\bar{g}}_{g_{1}}=0$, and $\widehat{\bar{g}}_{g_{2}}=0$. In addition, the design parameters are chosen as $k_{1}=$ $2, k_{2}=1, \gamma_{\theta_{1}}=\gamma_{\theta_{2}}=2, \gamma_{b_{1}}=\gamma_{b_{2}}=3, \gamma_{\bar{g}_{1}}=\gamma_{\bar{g}_{2}}=4, \omega_{1}=$ $\omega_{2}=\mu_{1}=\mu_{2}=\nu_{1}=\nu_{2}=0.0001, \omega=0.01$, and $\tau_{1}=0.1$. The Nussbaum function is chosen as $N(\zeta)=\zeta^{2} \cos (\zeta)$ with $\zeta(0)=0$. The initial states of $(53)$ are chosen as $x_{1}(0)=0$, $x_{2}(0)=0.5$.

The simulation results are shown in Figures 4, 5, 6, 7, 8 , and 9. From Figure 4, it is observed that the good tracking performance is achieved under the proposed approach.
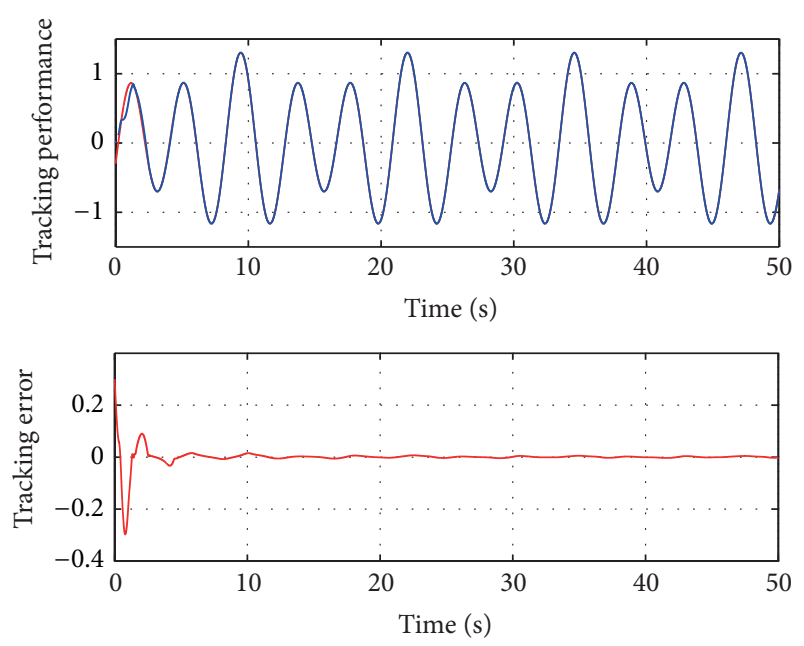

FIgURE 4: Tracking performance of the closed-loop system.

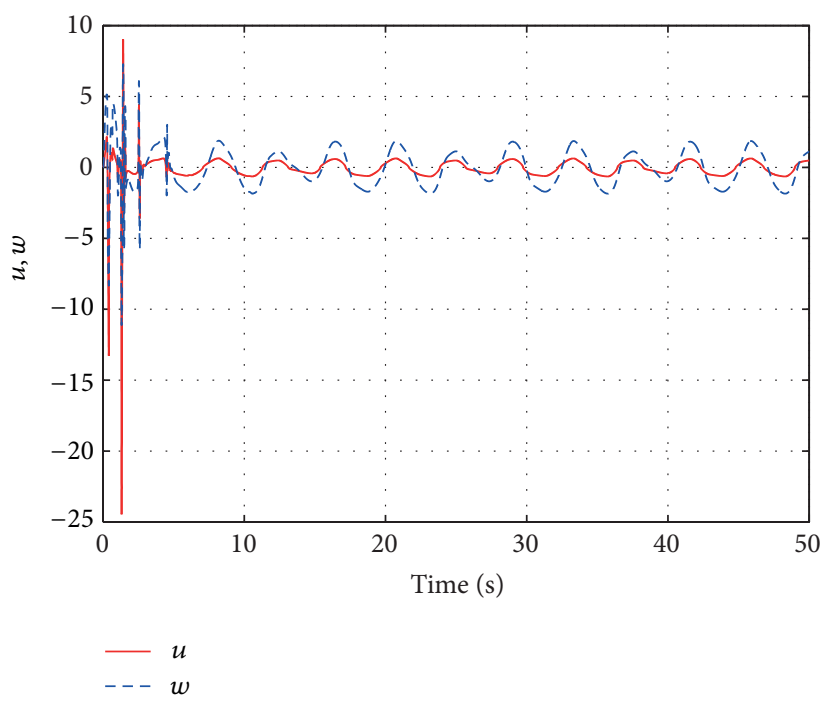

FIGURE 5: Control input $u$ (real line) and hysteresis output $w$ (dashed line).

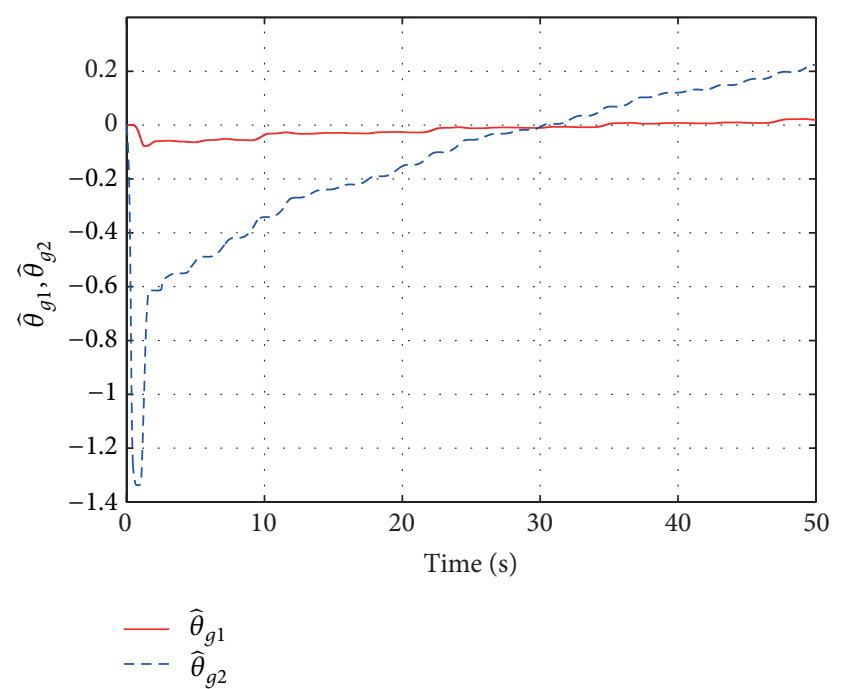

FIGURE 6: $\widehat{\theta}_{g_{1}}$ (real line) and $\widehat{\theta}_{g_{2}}$ (dashed line). 


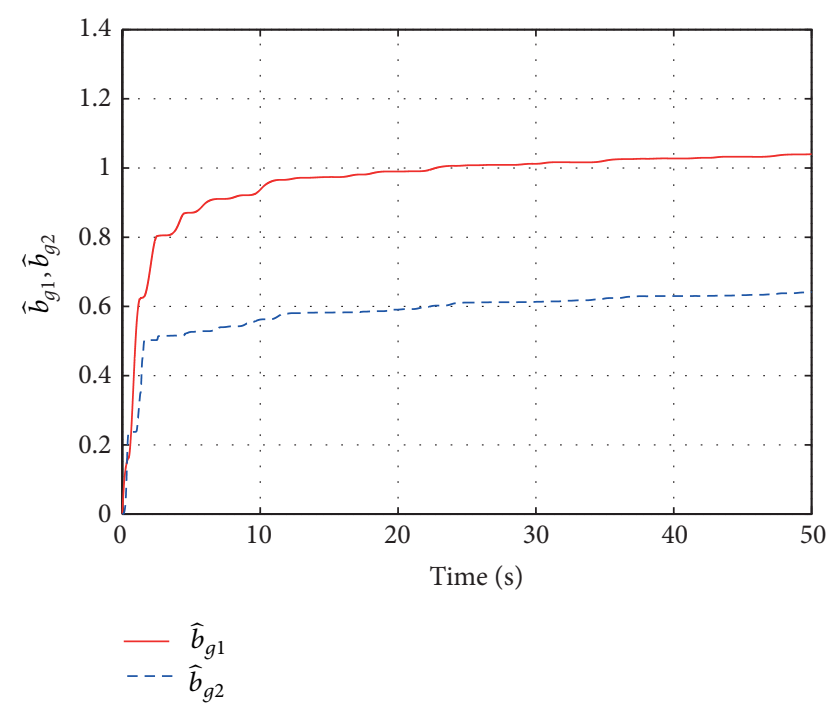

FIGURE $7: \widehat{b}_{g_{1}}$ (real line) and $\widehat{b}_{g_{2}}$ (dashed line).

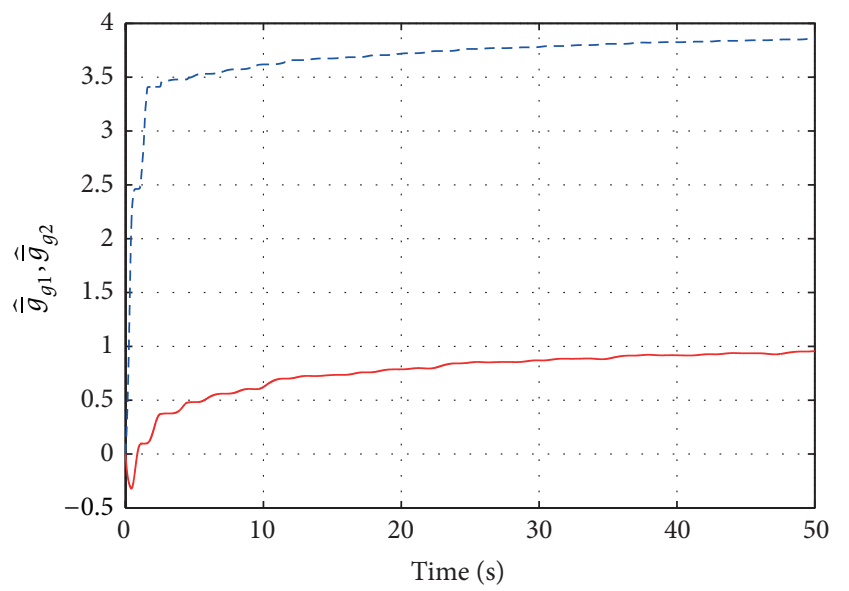

$-\hat{\bar{g}}_{g 1}$
$---\hat{\bar{g}}_{g 2}$

Figure 8: $\widehat{\bar{g}}_{g_{1}}$ (real line) and $\widehat{\bar{g}}_{g_{2}}$ (dashed line).

Figure 5 shows the control input $u$ and the hysteresis output $w$. Figures $6,7,8$, and 9 show the response curves of adaptive parameters $\widehat{\theta}_{g_{1}}, \widehat{\theta}_{g_{2}}, \widehat{b}_{g_{1}}, \widehat{b}_{g_{2}}, \widehat{\bar{g}}_{g_{1}}, \widehat{\bar{g}}_{g_{2}}$, and $\zeta$. From these results, the proposed scheme can mitigate the detrimental effects of the unknown hysteresis and guarantee the boundedness of the closed-loop system.

\section{Conclusion}

In this paper, the adaptive DSC approach for a class of uncertain nonlinear systems in strict-feedback form with unknown Duhem hysteresis is discussed. How to utilize the properties of the hysteresis model and design the related control approach is the main task for this topic. To overcome the design difficulties of Duhem model, three conditions are

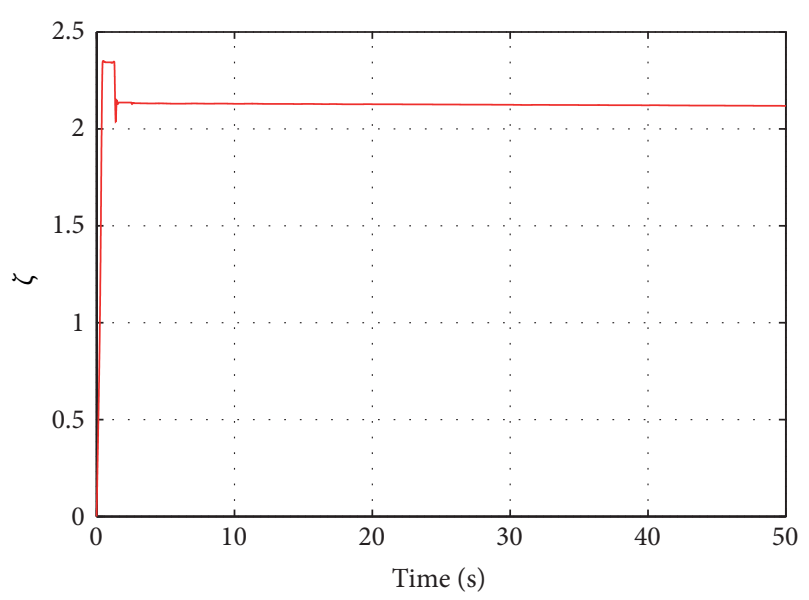

FIGURE 9: Variable $\zeta$.

used to get the analytical output expression of Duhem model. By using DSC technique, the "explosion complexity" in the standard backstepping design procedure is improved. For the last recursive step arising from the unknown hysteresis, the nonlinear smooth term of Duhem model is considered in the robust controller design by using mean value theorem and Nussbaum function lemma. Under the proposed approach, all the signals in the closed-loop system are uniformly semiglobally bounded, and a numerical example is shown to verify the effectiveness.

\section{Conflict of Interests}

The authors declare that there is no conflict of interests regarding the publication of this paper.

\section{Acknowledgments}

The work was partially supported by the Funds for Natural Science Foundation of China under Grants 61074097, 61105081, 61228301, and U1201244, the Program of Pearl River Young Talents of Science and Technology in Guangzhou (2013J2200100), the Integration of Industry, Education, and Research of Guangdong Province (2012B091100039).

\section{References}

[1] Y. Cao, L. Cheng, X. B. Chen, and J. Y. Peng, "An Inversionbased model predictive control with an integral-of-error state variable for Piezoelectric actuators," IEEE/ASME Transactions on Mechatronics, vol. 18, no. 3, pp. 895-904, 2013.

[2] Z. Li and C.-Y. Su, "Neural-adaptive control of single-master multiple slaves teleoperation for coordinated multiple mobile manipulators with time-varying communication delays and input uncertainty," IEEE Transactions on Neural Networks and Learning Systems, vol. 24, no. 9, pp. 1400-1413, 2013.

[3] Z. Li, X. Cao, Y. Yang, R. Li, and W. Ye, "Bilateral teleoperation of holonomic constrained robotic system with time-varying delays," IEEE Transactions on Instrumentation and Measurement, vol. 62, no. 4, pp. 752-765, 2013. 
[4] Z. Li, C. Yang, N. Ding, S. Bogdan, and T. Ge, "Robust adaptive motion control for remotely operated vehicles with velocity constraints," International Journal of Control, Automation, and System, vol. 10, no. 2, pp. 421-429, 2012.

[5] Z. Li, B. Wang, C. Yang, Q. Xie, and C. Y. Su, "Boosting-based EMG patterns classification scheme for robustness enhancement," IEEE Journal of Biomedical and Health Informatics, vol. 17, no. 3, pp. 545-552, 2013.

[6] I. Mayergoyz, Mathematical Models of Hysteresis and Their Applications, Elsevier, New York, NY, USA, 2003.

[7] M. Nordin and P. Gutman, "Controlling mechanical systems with backlash-a survey," Automatica, vol. 38, no. 10, pp. 16331649, 2002.

[8] G. V. Webb, D. C. Lagoudas, and A. J. Kurdila, "Hysteresis modeling of SMA actuators for control applications," Journal of Intelligent Material Systems and Structures, vol. 9, no. 6, pp. 432448, 1998.

[9] C.-Y. Su, Y. Stepanenko, J. Svoboda, and T. P. Leung, "Robust adaptive control of a class of nonlinear systems with unknown backlash-like hysteresis," IEEE Transactions on Automatic Control, vol. 45, no. 12, pp. 2427-2432, 2000.

[10] B. D. Coleman and M. L. Hodgdon, "A constitutive relation for rate-independent hysteresis in ferromagnetically soft materials," International Journal of Engineering Science, vol. 24, no. 6, pp. 897-919, 1986.

[11] Y. K. Wen, "Method for random vibration of hysteretic systems," Journal of Engineering Mechanics, vol. 102, no. 2, pp. 249-263, 1976.

[12] P. Duhem, "Die dauernden Aenderungen und die Thermodynamik," Zeitschrift für Physikalische Chemie, vol. 22, pp. 543589, 1897.

[13] M. A. Krasnoskl'skii and A. V. Pokrovskii, Systems with Hysteresis, Springer, New York, 1989.

[14] J. W. Macki, P. Nistri, and P. Zecca, "Mathematical models for hysteresis," SIAM Review, vol. 35, no. 1, pp. 94-123, 1993.

[15] F. Preisach, "Über die magnetische Nachwirkung," Zeitschrift für Physik, vol. 94, no. 5-6, pp. 277-302, 1935.

[16] S. A. Belbas and I. D. Mayergoyz, "Optimal control of dynamical systems with Preisach hysteresis," International Journal of NonLinear Mechanics, vol. 37, no. 8, pp. 1351-1361, 2002.

[17] Y. Feng, Y. Hu, C. A. Rabbath, and C. Su, "Robust adaptive control for a class of perturbed strict-feedback non-linear systems with unknown Prandtl-Ishlinskii hysteresis," International Journal of Control, vol. 81, no. 11, pp. 1699-1708, 2008.

[18] A. Gudovich and M. Quincampoix, "Optimal control with hysteresis nonlinearity and multidimensional play operator," SIAM Journal on Control and Optimization, vol. 49, no. 2, pp. 788-807, 2011.

[19] C.-Y. Su, Y. Feng, H. Hong, and X. Chen, "Adaptive control of system involving complex hysteretic nonlinearities: a generalised Prandtl-Ishlinskii modelling approach," International Journal of Control, vol. 82, no. 10, pp. 1786-1793, 2009.

[20] P. Krejci and K. Kuhnen, "Inverse control of systems with hysteresis and creep," IEE Proceedings, vol. 148, no. 3, pp. 185192, 2001.

[21] G. Tao and P. V. Kokotovic, "Adaptive control of plants with unknown hystereses," IEEE Transactions on Automatic Control, vol. 40, no. 2, pp. 200-212, 1995.

[22] F. Ikhouane, V. Mañosa, and J. Rodellar, "Adaptive control of a hysteretic structural system," Automatica, vol. 41, no. 2, pp. 225231, 2005.
[23] C.-Y. Su, Q. Wang, X. Chen, and S. Rakheja, "Adaptive variable structure control of a class of nonlinear systems with unknown Prandtl-Ishlinskii hysteresis," IEEE Transactions on Automatic Control, vol. 50, no. 12, pp. 2069-2074, 2005.

[24] J. Zhou, C.-Y. Wen, and Y. Zhang, "Adaptive backstepping control of a class of uncertain nonlinear systems with unknown backlash-like hysteresis," IEEE Transactions on Automatic Control, vol. 49, no. 10, pp. 1751-1757, 2004.

[25] Y. Feng, C. A. Rabbath, T. Chai, and C.-Y. Su, "Robust adaptive control of systems with hysteretic nonlinearities: a Duhem hysteresis modelling approach," in Proceedings of the IEEE Africon 2009, pp. 1-6, New York, NY, USA, September 2009.

[26] D. Swaroop, J. K. Hedrick, P. P. Yip, and J. C. Gerdes, "Dynamic surface control for a class of nonlinear systems," IEEE Transactions on Automatic Control, vol. 45, no. 10, pp. 1893-1899, 2000.

[27] M. L. Hodgdon, "Applications of a theory of ferromagnetic hysteresis," IEEE Transactions on Magnetics, vol. 24, no. 1, pp. 218-221, 1987.

[28] M. L. Hodgdon, "Mathematical theory and calculations of magnetic hysteresis curves," IEEE Transactions on Magnetics, vol. 24, no. 6, pp. 3120-3122, 1988.

[29] S. S. Ge, F. Hong, and T. H. Lee, "Adaptive neural control of nonlinear time-delay systems with unknown virtual control coefficients," IEEE Transactions on Systems, Man, and Cybernetics B, vol. 34, no. 1, pp. 499-516, 2004.

[30] M. M. Polycarpou and P. A. Ioannou, "A robust adaptive nonlinear control design," Automatica, vol. 32, no. 3, pp. 423427, 1996.

[31] E. P. Ryan, "A universal adaptive stabilizer for a class of nonlinear systems," Systems and Control Letters, vol. 16, no. 3, pp. 209-218, 1991. 


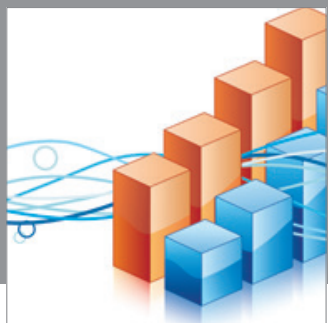

Advances in

Operations Research

mansans

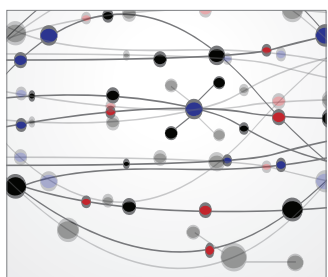

The Scientific World Journal
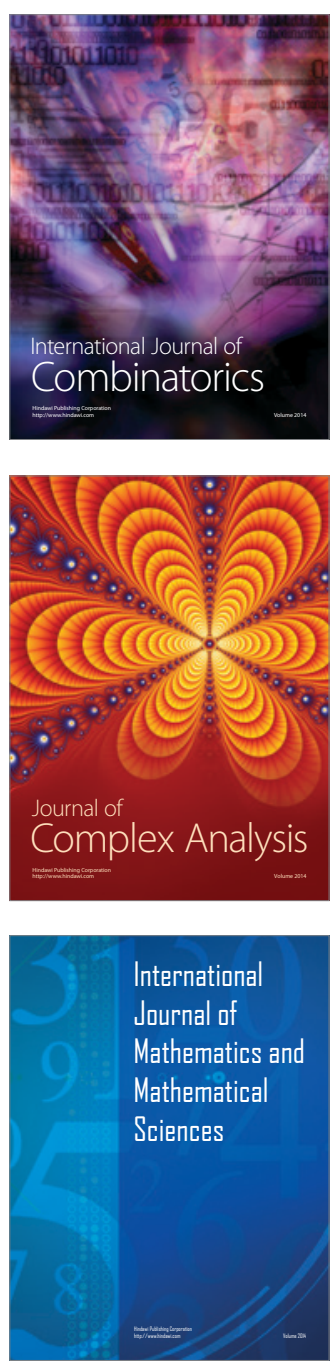
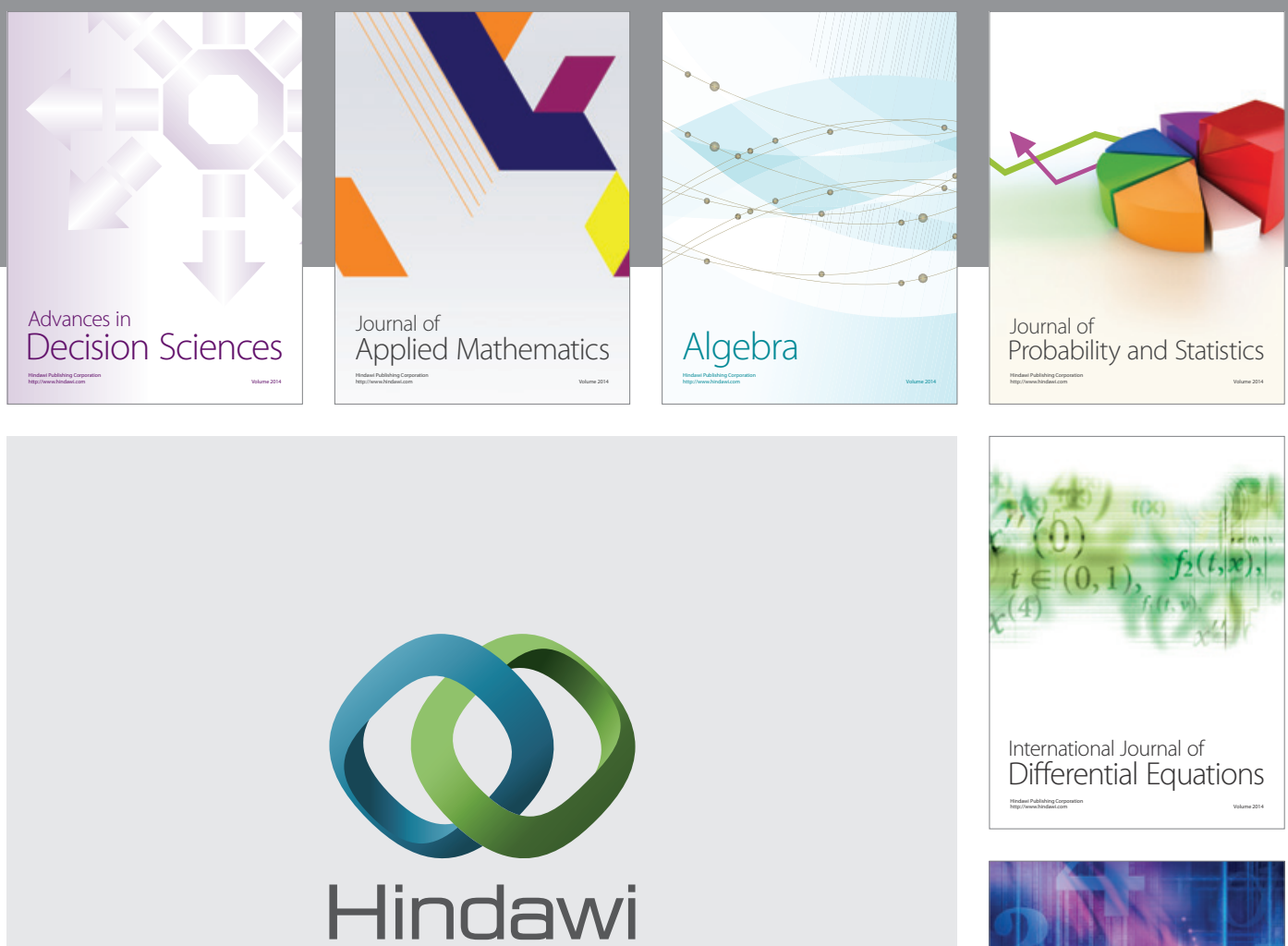

Submit your manuscripts at http://www.hindawi.com
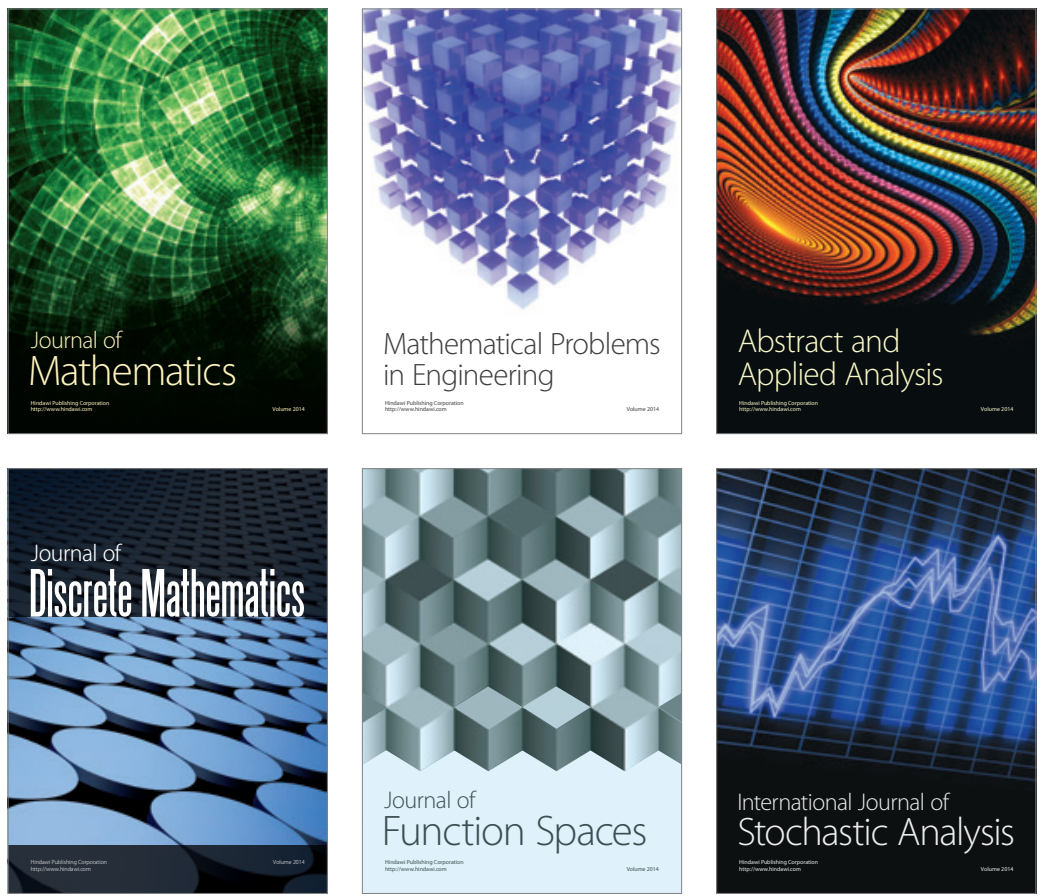

Journal of

Function Spaces

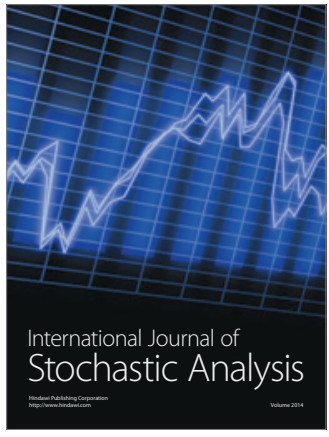

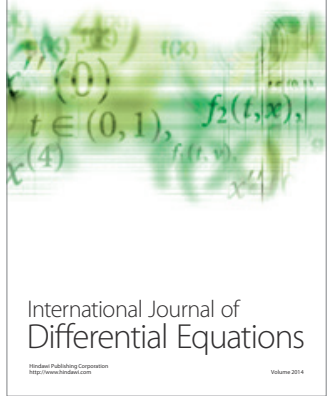
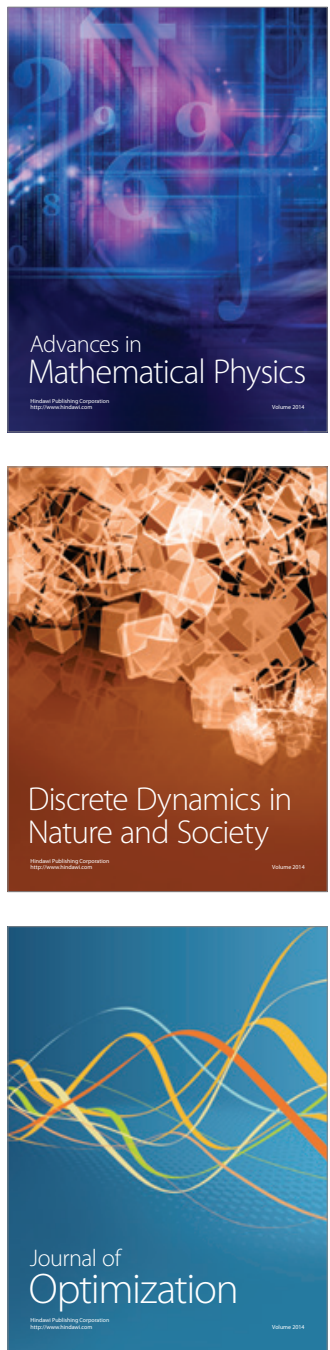